\title{
From E-VITA Open Plus to E-VITA NEO and E-NOVIA
}

\author{
Heinz Jakob ${ }^{1}$, Mohammed Idhrees ${ }^{2}$, and Mohamad Bashir ${ }^{3}$ \\ ${ }^{1}$ West German Heart Centre Essen \\ ${ }^{2}$ SRM Institutes for Medical Science Vadapalani \\ ${ }^{3}$ Royal Blackburn Teaching Hospital
}

August 28, 2020

\begin{abstract}
Abstract The first clinical implantation of the "Essen I prosthesis" took place in 2005, which was then followed by E-Vita open plus. With further advancements E-Vita Neo and E-Novia was introduced. These devices enable the surgeons to perform FET in zone 0/1 which eventually reduce the incidence of paraplegia, recurrent laryngeal nerve palsy and proximalization of supraaortic arch vessels. E-vita open plus and successors alleviate frozen elephant trunk operations rendering more stable results in promoting positive remodelling of the distal aorta.
\end{abstract}

\section{Introduction}

The frozen elephant trunk (FET) facilitates access to the ascending aorta, aortic arch, and the descending thoracic aorta via a median sternotomy, helping to address a wide range of aortic pathology. With increasing in popularity, over 30,000 hybrid prosthesis has been implanted across the globe [1]. The understanding of aortic pathology and the pathophysiological mechanisms coupled with the advancements in the techniques and technology has produced excellent long term results of survival probability of 5 years for acute Type A aortic dissection, chronic aortic dissection and thoracic aortic aneurysm at $91 \%, 98 \%$ and $92 \%$ respectively [2].

\section{Evolution of the FET}

Started as "Essen I Prosthesis", the hybrid prosthesis was first commercially available as Evita Open (Jotec $\AA$, Hechingen, Germany) in 2005 [3]. With the non-availability of the hybrid prosthesis in USA, surgeons have explored different options - antegrade delivery of a stent graft during deep hypothermic circulatory arrest (DHCA)[4] or by creating a scallop in the endoprosthesis to accommodate the supra aortic arch vessels [5]. A major concern of FET was the higher incidence of paraplegia compared to the classical elephant trunk. This prompted the surgeons to reduce the length of the stent-graft and / or proximalize the distal anastomosis from zone 3 to zone 2 [6]. This further paved the way for technical advancements wherein surgeons introduced a fenestration of the stent graft for the left subclavian artery (LSA) [7] or a side branch to the LSA in the hybrid prosthesis. Sometimes, surgical efforts to reimplant the very distal and posterior left subclavian artery included the intra-thoracic aortic-subclavian bypass, aortic-axillary bypass and extra-thoracic carotid-subclavian bypass [8]. This warrants an additional incision in the left infraclavicular or supraclavicular region. These advancements entails the adjustments of the extracorporeal circuit [6].Though with the advancements, the FET still requires DHCA with selective antegrade cerebral circulation (SACP), restricting the procedure to be performed by surgeons familiar with aortic arch surgery. To overcome this, newer technical devices developed by JOTEC/CryoLife, Kennesaw, USA are being introduced aiming to reduce complexity enabling surgeons to perform FET in zone 0 or 1 as opposed to zone 3 (Fig.1)

Proximalization to zone 0/1- The further we advance 
Though the FET has recently been implanted in zone 2, it is not without challenges and complications. These challenges and complications may be less as compared to implantation in zone3, but they still persists - recurrent laryngeal nerve palsy $(2.8 \%$ vs $5.2 \%, \mathrm{p}=0.526)$, permanent neurological defect $(5.8 \%$ vs $9.9 \%$, $\mathrm{p}=0.431$ ), paraplegia ( 0 vs $4.7 \%, \mathrm{p}=0.191$ ), bleeding ( $15.9 \%$ vs $12.2 \%, \mathrm{p}=0.553$ ) [9]. In the quest to reduce the complication, newer technical devices are being introduced. This necessitates the debranching of the supra-aortic arch vessels to zone 0 and later implant the hybrid prosthesis in zone $0 / 1$. Different techniques to debranch the three supra-aortic vessels in the mediastinum to Zone 0 are described - trifurcation arch graft with a perfusion side arm port (TAPP graft, Vascutek Ltd., Renfrewshire, Scotland [10], 4-branched Dacron graft [11], Lupiae prosthesis (Vascutek Terumo Inc, Scotland, UK) [12] or individual branch graft to each branch vessel [13]. Among these 'branch first' techniques popularized by Matalanis has a minimum DHCA time and reduced cardiopulmonary bypass time [10]. With the advent of the neo E-vita and E-novia, the hybrid prosthesis can be implanted in zone 0 to 3 , and the supra aortic arch vessels can be reimplanted individually, en bloc or just left in situ with an uncovered stent graft

The advantages to implant the graft in zone $0 / 1$ include (a) proximalization of aortic arch to zone 0 (b) surgeons not familiar with aortic arch and antegrade circulation can as well perform the procedure with a brief period of DHCA after initial debranching (c) Can eliminate the incidence of recurrent laryngeal nerve palsy and significantly decrease paraplegia (d) The techniques allow a systematic interrogation of each branch anastomosis ensuring secure hemostatic anastomosis (e) Distal anastomosis is more proximal, enabling an easier hemostatic check after the release of cross clamp.

Patients, who are sick with complex and very acute aortic dissection including distal arch/proximal descending aorta re-entry tears, are not suitable for total arch replacement or any complex surgery like FET as a prolonged cardiopulmonary bypass time may be deleterious. These patients may benefit from E-novia. The hybrid prosthesis consists of a distal covered and proximal non-covered stent-graft portion. The covered stent-graft is placed in the descending thoracic aorta while the non-covered stent-graft is accommodated in the aortic arch. The proximal anastomosis would be performed in Zone 0, eventually reducing the ischemic time and hypothermic circulatory arrest time. This combines the classic fast proximal aortic repair with the proposed downstream benefit of the descending aorta in FET. The concept is similar to the PETTICOAT experience in acute type B dissection where the branch vessel stays open when there is adequate run off in the target vessel. The early result in 6 patients has been published recently [14], and discussed by Roselli [15]. These are at present reserved for acute type I aortic dissection, Penn B, C, BC, and patients with severe concomitant disease [5].

\section{Acute Aortic Dissection}

In the era of E-VITA Open Plus the supra aortic arch vessels were reimplanted enbloc. This will require an additional Foley's catheter inserted into the hybrid prosthesis to perfuse the lower body. This can be cumbersome. This is made more simplified with the E-vita Neo which has a side branch to perfuse the lower body before the reimplantation of the supra aortic arch vessel (Fig.1). In very sick and acute aortic dissection, E-NOVIA can be implanted in zone 0 as described above.

\section{Chronic Aortic dissection (CAD)}

These are usually hemodynamically stable patients. The most preferred option at present is zone 2 implantation of hybrid prosthesis. With the advent of the E-VITA Open Plus, it is possible to perform a zone 0 implantation in these patients as well. The supra-aortic arch vessels can be debranched in a way similar to Matalanis and team [10]. These debranched vessels can then be incorporated into the hybrid prosthesis. This is easier and faster than the conventional FET in zone 2.

\section{Thoracic aortic aneurysm}

Surgical treatment of patients with multisegmental aneurysms can be handled similarly to CAD patients. The size of the E-vita open plus or E-vita NEO is adjusted to the distal landing zone in the descending aorta with mild or no oversizing, whereas the arch is either totally removed or used as outer sleeve surrounding 
the proximal part to be anastomosed to the ascending aorta in zone 0 or 1 . In case of debranching of the head vessels, reimplantation can be done wherever appropriate.

\section{Conclusion}

E-vita open plus and successors alleviate frozen elephant trunk operations rendering more stable results in promoting positive remodelling of the distal aorta. In patients with aortic dissections the remodelling of aorta favor a long term freedom from reintervention or reoperation upto the segment of stent graft. [16] (Fig. 2). Livelong surveillance is required to detect the development of downstream pathologies due to persistent type III dissection with false lumen growth either after acute or chronic dissection (Fig. 2). In case of thoracoabdominal aneurysms, where a planned second stage intervention or operation is warranted, endovascular treatment and even open surgery is facilitated due to the midthoracic docking station within the distal part of the E-vita stent grafts.

\section{References}

1. Ma WG, Zheng J, Sun LZ, Elefteriades JA. Open stented grafts for frozen elephant trunk technique: technical aspects and current outcomes. Aorta (Stamford) 2015;3:122-35.

2. Jakob H, Moughal S, Bashir M. Frozen elephant trunk with straight vascular prosthesis: single-center experience with a review of current trends. J Cardiovasc Surg 2020;61:301-7. DOI: 10.23736/S0021-9509.20.114010 .

3. Jakob H, Tsagakis K, Leyh R, et al. Development of an integrated stent graft-dacron prosthesis for intended one-stage repair in complex thoracic aortic disease. Herz 2005;30:766-8

4. Pochettino A, Brinkman WT, Moeller P, Szeto WY, Moser W, Cornelius K et al. Antegrade thoracic stent grafting during repair of acute DeBakey I dissection prevents development of thoracoabdominal aortic aneurysms. Ann Thorac Surg 2009;88:482-9.

5. Roselli EE, Idrees JJ, Bakaeen FG, Tong MZ, Soltesz EG, Mick S et al. Evolution of simplified frozen elephant trunk repair for acute DeBakey type I dissection: midterm outcomes. Ann Thorac Surg 2018; 105:749-55.

6. Jakob H, Idhrees M, Bashir M. Frozen elephant trunk with straight vascular prosthesis. Ann Cardiothorac Surg . 2020;9(3):164-169. doi:10.21037/acs-2020-fet-60

7. Roselli EE, Tong MZ, Bakaeen FG. Frozen elephant trunk for DeBakey type 1 dissection: the Cleveland Clinic technique. Ann Cardiothorac Surg 2016;5:251-5.

8. Tsagakis K, Dohle DS, Wendt D, et al. Left subclavian artery rerouting and selective perfusion management in frozen elephant trunk surgery. Minim Invasive Ther Allied Technol 2015;24:311-6

9. Leone A, Di Marco L, Coppola G, et al. Open distal anastomosis in the frozen elephant trunk technique: initial experiences and preliminary results of arch zone 2 versus arch zone $3+$. Eur J Cardiothorac Surg . 2019;56(3):564-571. doi:10.1093/ejcts/ezz10.

10. Matalanis G, Galvin SD. "Branch-first" continuous perfusion aortic arch replacement and its role in intra-operative cerebral protection.Ann Cardiothorac Surg . 2013;2(2):194-201. doi:10.3978/j.issn.2225319X.2013.02.01

11. Bavaria J, Milewski RK, Baker J, Moeller P, Szeto W, Pochettino A. Classic hybrid evolving approach to distal arch aneurysms: toward the zone zero solution. J Thorac Cardiovasc Surg . 2010;140(6 Suppl):S77S91. doi:10.1016/j.jtcvs.2010.07.031

12. Marullo AG, Bichi S, Pennetta RA, et al. Hybrid aortic arch debranching with staged endovascular completion in DeBakey type I aortic dissection. Ann Thorac Surg . 2010;90(6):1847-1853. doi:10.1016/j.athoracsur.2010.07.077 
13. Idhrees, M., Krishnaswami, M., Jacob, A. et al. Hybrid aortic arch repair: 10-year experience from India. Indian J Thorac Cardiovasc Surg 35, 156-163 (2019). https://doi.org/10.1007/s12055-018-0689-0.

14. Jakob H, Shehada SE, Dohle D, et al. New 3-zone hybrid graft: First-in-man experience in acute type I dissection [published online ahead of print, 2020 May 6]. J Thorac Cardiovasc Surg . 2020;S00225223(20)31080-1. doi:10.1016/j.jtcvs.2020.04.113

15. Roselli E. Commentary: Embracing the future by bracing the archdoi.org/10.1016/j.jtcvs.2020.05.037

16. Dohle DS, Tsagakis K, Janosi RA, et al. Aortic remodelling in aortic dissection after frozen elephant trunk+. Eur J Cardiothorac Surg . 2016;49(1):111-117. doi:10.1093/ejcts/ezv045

\section{Legends}

Figure 1: The application of variations of E-vita NEO and E-Novia are being demonstrated in regards to Ishimaru's Aortic arch zone.

Figure 2: Essen- Complete thrombosis results 1 year after surgery: Percentage of complete thrombosis + obliteration of false lumen/aneurysm in acute aortic dissection (AD), chronic AD, and thoracic aortic aneurysm (TAA) in descening aorta (Thoracic / Abdominal) 

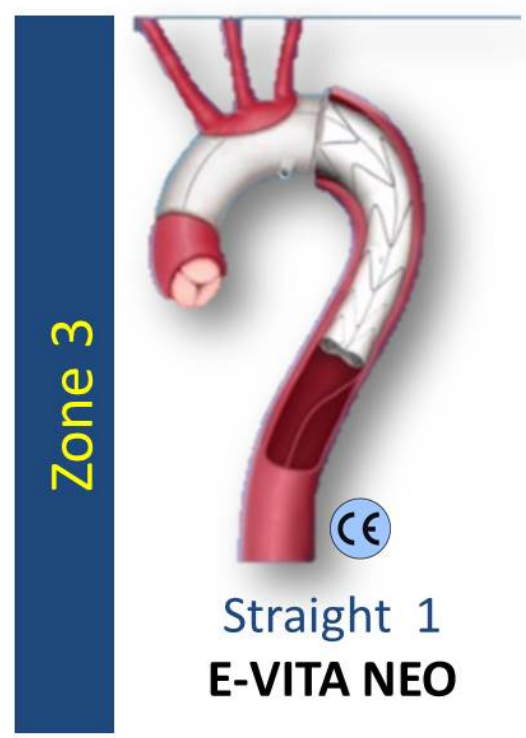

Straight 1

\section{E-VITA NEO}

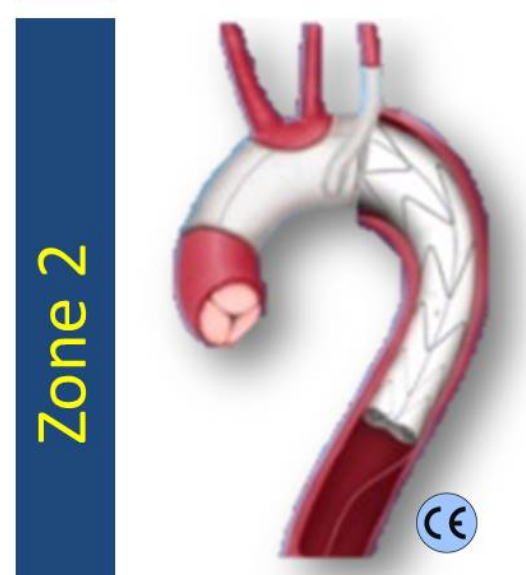

Straight 2

E-VITA NEO
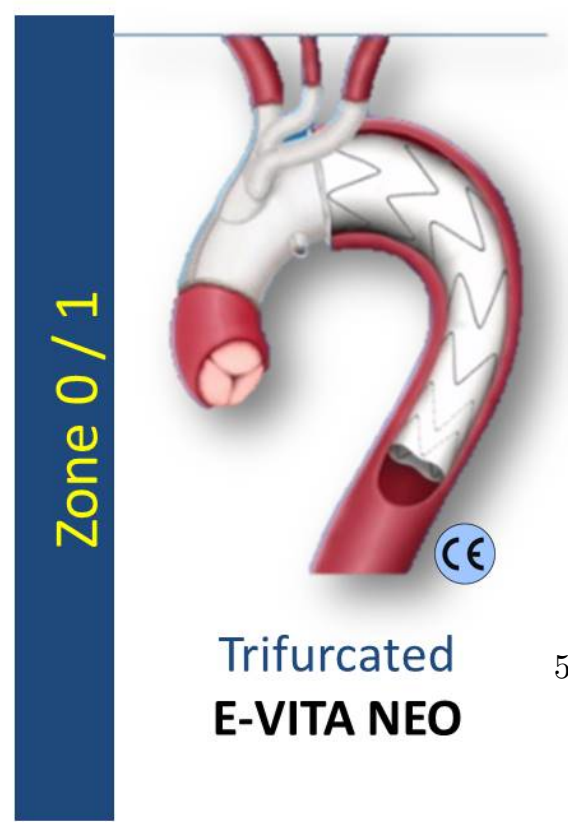

Trifurcated

E-VITA NEO

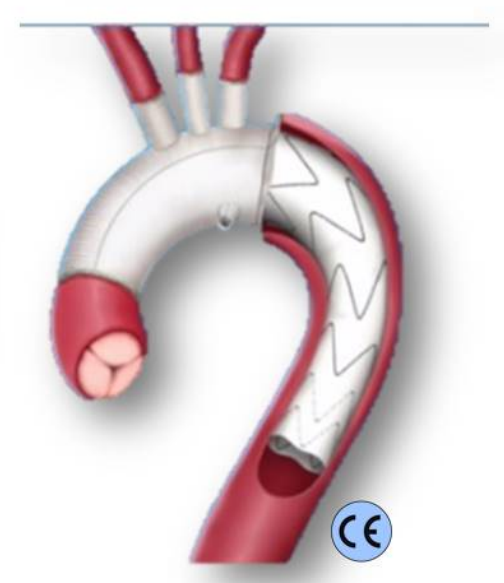

Branched

E-VITA NEO

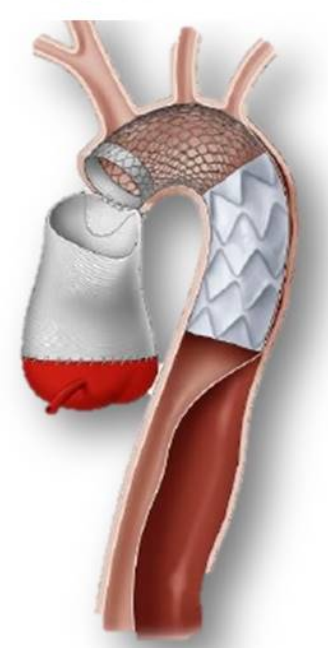

E-NOVIA 


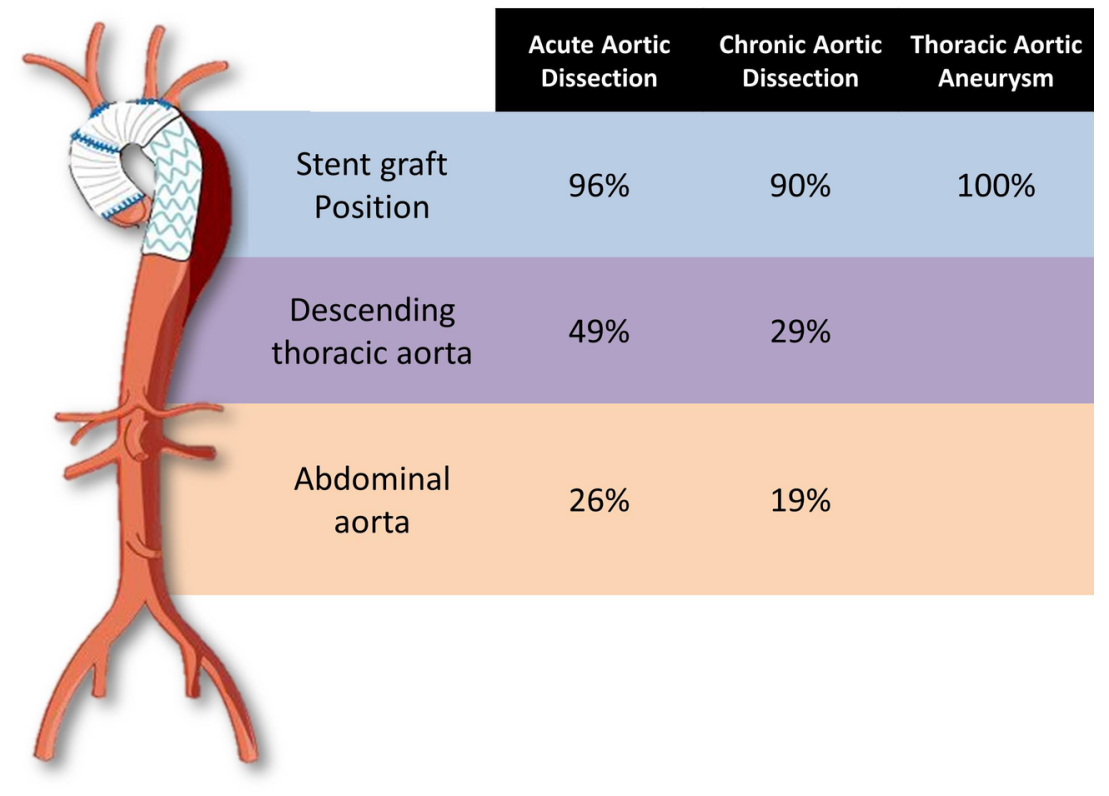

